\title{
Fixed Point Theorem for Multivalued Non-self Mappings in Partial Symmetric Spaces
}

https://doi.org/10.1515/taa-2021-0102

Received 19 May, 2021; revised 15 July, 2021; accepted 30 July, 2021

Abstract: In this paper, we proved a fixed point theorem for multi-valued non-self mappings in partial symmetric spaces. In doing so, we extended and generalized the results in literature by employing a convex structure for multi-valued non-self mappings using Rhoades type contractions. We also provided an illustrative example to support the results.

Keywords: Fixed point, partial symmetric spaces, multi-valued mapping, non-self mappings.

\section{Introduction}

In 1922, Banach [1] gave a contraction principle (BCP) for a self-mapping in metric space. Later on, Nadler [2] extended the Banach contraction principle from a single-valued map into the multi-valued map. Assad and Kirk [3] extended the results of Nadler to the subset of metrically convex metric space with Rothe's boundary condition to obtain the fixed point. Cirić [4] gave a generalized Banach contraction principle and proved fixed point theorems for single-valued and multi-valued quasi-contractions.

Ćirić [5] proved a fixed point theorem for a class of non-self mappings that satisfy a generalized contraction condition. Several researchers proved different results for non-self contractive mappings. One can see, e.g., [6-10].

Fixed point theory for non-self multi-valued mappings was developed rapidly after the publication of Assad and Kirk's paper [3] in which they proved a non-self multi-valued version of Banach's contraction principle. Rhoades [11, 12] obtained the fixed point theorem for non-self set-valued mappings satisfying contractive type conditions. Du et al. [13] proved fixed point theorems for various multi-valued non-self maps by extending and generalizing self-maps to the class of non-self maps. For more results on multi-valued non-self maps, we refer the reader in [14-18] and the references therein.

In 2019, Asim et al. [19] initiated the study of Partial symmetric space by combining the concept of partial metric space due to Matthew [20] and symmetric space concept due to Wilson [21]. Using this space, they proved some related fixed point results for single-valued and multi-valued mappings. Since then, several researchers getting motivation to do their research in this direction. In 2021, Asim and Imdad [22] proved a common fixed point result in partial symmetric space. Furthermore, Asim et al. [23] proved a multi-valued result using Suzuki and Wardowski-type contraction mapping in partial symmetric space.

In this paper, we attempted to use the Rhoades contraction method to generalize the theorem due to Assad, and Kirk [3] for multi-valued non-self mappings on partial symmetric space. With this, we have denoted $\left(X, p_{s}\right)$ as a partial symmetric space.

Lucas Wangwe: Department of Mathematics, College of Natural and Applied Sciences, University of Dar es Salaam, Tanzania; E-mail: wangwelucas@gmail.com

*Corresponding Author: Santosh Kumar: Department of Mathematics, College of Natural and Applied Sciences, University of Dar es Salaam, Tanzania; E-mail: drsengar2002@gmail.com 


\section{Preliminaries}

The following preliminaries and results will be helpful to develop the new theorem for this paper.

Definition 2.1. [19] Let $X$ be a non-empty set. A mapping $p_{s}: X \times X \rightarrow \mathbb{R}_{+}$is said to be partial symmetric if, for all $x, y, z \in X$, we have the following properties:

$\left(p_{s 1}\right): x=y$ if and only if $p_{s}(x, y)=p_{s}(x, x)=p_{s}(y, y)$,

$\left(p_{s 2}\right): p_{s}(x, x) \leq p_{s}(x, y)$,

$\left(p_{s 3}\right): p_{s}(x, y)=p_{s}(y, x)$.

Then the pair $\left(X, p_{s}\right)$ is said to be partial symmetric space.

From $p_{s 1}$ and $p_{s 2}$ we have

$$
p_{s}(x, y)=0 \Rightarrow p_{s}(x, y)=p_{s}(x, x)=p_{s}(y, y) \Rightarrow x=y .
$$

A partial symmetric space $\left(X, p_{s}\right)$ reduces to a symmetric space if $p_{s}(x, x)=0$, for all $x \in X$. Obviously, every symmetric space is partial symmetric space, but not conversely.

Let $\left(X, p_{s}\right)$ be a partial symmetric space. Then, the $p_{s}$-open ball, with center $x \in X$ and radius $\varepsilon>0$, is defined by: $B_{p_{s}}(x, \varepsilon)=\left\{y \in X: p_{s}(x, y)<p_{s}(x, x)+\varepsilon\right\}$,

Similarly, the $p_{s}$-closed ball, with center $x \in X$ and radius $\varepsilon>0$, is defined by: $B_{p_{s}}[x, \varepsilon]=\{y \in X$ : $\left.p_{s}(x, y) \leq(x, x)+\varepsilon\right\}$.

The family of $p_{s}$-open balls for all $x \in X$ and $\varepsilon>0, U_{p_{s}}=\left\{B_{p_{s}}(x, \varepsilon): x \in X, \varepsilon>0\right\}$, forms basis of some topology $\tau_{p_{s}}$ on $X$.

Definition 2.2. [19] Let $\left(X, p_{s}\right)$ be a partial symmetric space. Then,

(i) a sequence $\left\{x_{n}\right\}$ in $\left(X, p_{s}\right)$ is said to be $p_{s}$-convergent to $x \in X$, with respect to $\tau_{p_{s}}$ if $p_{s}(x, x)=\lim _{n \rightarrow \infty} p_{s}\left(x_{n}, x\right)$.

(ii) a sequence $\left\{x_{n}\right\}$ in $\left(X, p_{s}\right)$ is called a $p_{s}$-Cauchy sequence if only if $\lim _{n, m \rightarrow \infty} p_{s}\left(x_{n}, x_{m}\right)$ exists and is finite.

(iii) a partial symmetric space $\left(X, p_{s}\right)$ is said to be $p_{s^{-}}$-complete if every $p_{s^{-}}$Cauchy sequence $\left\{x_{n}\right\}$ in $X$ is $p_{s}$ convergent, with respect to $\tau_{p_{s}}$ to a point $x \in X$, such that

$$
p_{s}(x, x)=\lim _{n \rightarrow \infty} p_{s}\left(x_{n}, x\right)=\lim _{n, m \rightarrow \infty} p_{s}\left(x_{n}, x_{m}\right) .
$$

Definition 2.3. [19] Let $\left(X, p_{s}\right)$ be a partial symmetric space. Then

$\left(A_{1}\right) \lim _{n \rightarrow \infty} p_{s}\left(x_{n}, x\right)=p_{s}(x, x)$ and $\lim _{n \rightarrow \infty} p_{s}\left(x_{n}, y\right)=p_{s}(x, y)$ imply that $x=y$, for a sequence $\left\{x_{n}\right\}, x, y \in X$.

$\left(A_{2}\right)$ a partial symmetric $p_{s}$ is said to be 1-continuous if $\lim _{n \rightarrow \infty} p_{s}\left(x_{n}, x\right)=p_{s}(x, x)$ implies that $\lim _{n \rightarrow \infty} p_{s}\left(x_{n}, y\right)=$ $p_{s}(x, y)$, where $\left\{x_{n}\right\}$ is a sequence in $X$ and $x, y \in X$.

$\left(A_{3}\right)$ a partial symmetric $p_{s}$ is said to be continuous if $\lim _{n \rightarrow \infty} p_{s}\left(x_{n}, x\right)=p_{s}(x, x)$ and $\lim _{n \rightarrow \infty} p_{s}\left(x_{n}, y\right)=p_{s}(x, y)$ imply that $\lim _{n \rightarrow \infty} p_{s}\left(x_{n}, y_{n}\right)=p_{s}(x, y)$ where $\left\{x_{n}\right\}$ and $\left\{y_{n}\right\}$ are sequences in $X$ and $x, y \in X$.

$\left(A_{4}\right) \lim _{n \rightarrow \infty} p_{s}\left(x_{n}, x\right)=p_{s}(x, x)$ and $\lim _{n \rightarrow \infty} p_{s}\left(x_{n}, y_{n}\right)=p_{s}(x, x)$ imply $\lim _{n \rightarrow \infty} p_{s}\left(y_{n}, x\right)=p_{s}(x, x)$, for sequences $\left(x_{n}\right),\left(y_{n}\right)$, and $x$ in $X$.

$\left(A_{5}\right) \lim _{n \rightarrow \infty} p_{s}\left(x_{n}, y_{n}\right)=p_{s}(x, x)$ and $\lim _{n \rightarrow \infty} p_{s}\left(y_{n}, z_{n}\right)=p_{s}(x, x)$ imply $\lim _{n \rightarrow \infty} p_{s}\left(x_{n}, z_{n}\right)=p_{s}(x, x)$, for sequences $\left(x_{n}\right),\left(y_{n}\right),\left(z_{n}\right)$, and $x$ in $X$.

Definition 2.4. [19] Let $\left(X, p_{s}\right)$ be a partial symmetric space. A mapping $f: X \rightarrow X$ is said to be a Kannan-Ciric type $k$-contraction if, for all $x, y \in X$,

$$
p_{s}(f x, f y) \leq k \max \left\{p_{s}(x, f x), p_{s}(y, f y)\right\}
$$


where $k \in[0,1)$.

In 2019 Asim et al. [19], proved a Kannan- Ćirić type $k$ - contractions in the setting of partial symmetric space.

Theorem 2.1. [19] Let $\left(X, p_{s}\right)$ be a complete partial symmetric space and $f: X \longrightarrow X$. Assume that the following conditions are satisfied:

(i) $f$ is a Kannan- Cirić type $k$ - contraction,

(ii) $f$ is continuous.

Then $T$ has a unique fixed point $x \in X$ such that $p_{s}(x, x)=0$.

Example 1.1 [19] Let $X=\mathbb{R}$. Define a mapping $p_{s}: X \times X \rightarrow \mathbb{R}_{+}$for all $x, y \in X$ and $p, q>1$, as follows:

$$
p_{s}(x, y)=|x-y|^{p}+|x-y|^{q} .
$$

Then the pair $\left(X, p_{s}\right)$ is a partial symmetric space.

Example 1.2 [19] Let $X=\mathbb{R}$. Define a mapping $p_{s}: X \times X \rightarrow \mathbb{R}_{+}$for all $x, y \in X$ and $p, q>1$, as below:

$$
p_{s}(x, y)=(\max \{x, y\})^{p}+(\max \{x, y\})^{q} .
$$

Then the pair $\left(X, p_{s}\right)$ is a partial symmetric space.

Now, we are introducing some concepts of Hausdorff spaces as defined by Asim et al. [19] in partial symmetric spaces.

Let $\left(X, p_{s}\right)$ be partial symmetric space and $C B^{p s}(X)$ be the family of all nonempty, $\tau_{p s}$-closed and bounded subsets of $\left(X, p_{s}\right)$.

Observe that $A$ is said to be bounded if there exists $x \in X$ and $N \geq 0$, such that for all $a \in A$,

$$
p_{s}\left(x_{0}, a\right) \leq p_{s}(a, a)+N .
$$

Furthermore, for $A, B \in C B^{p_{s}}(X)$ and $x \in X$, we define:

$$
\begin{aligned}
\operatorname{dist}_{p_{s}}(x, A) & =\inf \left\{p_{s}(x, a): a \in A\right\} ; \\
\delta_{p_{s}}(A, B) & =\sup \left\{\operatorname{dist} p_{s}(a, B): a \in A\right\} ; \\
\delta_{p_{s}}(B, A) & =\sup \left\{\operatorname{dist} p_{s}(b, A): b \in B\right\} .
\end{aligned}
$$

Lemma 2.1. [19] Let $\left(X, p_{s}\right)$ be a partial symmetric space and $A$ a nonempty subset of $X$, then $a \in \bar{A}$ if and only if

$$
\operatorname{dist}_{p s}(a, A)=p_{s}(a, a),
$$

where $\bar{A}$ is $p$ - closed in $\left(X, p_{s}\right)$ iff $A=\bar{A}$.

Proposition 2.1. [19] Let $\left(X, p_{s}\right)$ be a complete partial symmetric space, for $A, B, C \in C B^{p_{s}}(X)$, then we have the following;

(i) $\delta_{p_{s}}(A, A)=\sup \left\{\operatorname{distp}_{s}(a, a): a \in A\right\}$;

(ii) $\delta_{p_{s}}(A, A)<\delta_{p_{s}}(A, B)$;

(iii) $B \subset C \Rightarrow \delta_{p_{s}}(A, C)<\delta_{p_{s}}(A, B)$;

(iv) $\delta_{p_{s}}(A, B)=0 \Rightarrow A \subseteq B$; and

(v) $\delta_{p_{s}}(A \cup B, C)=\max \left\{\delta_{p_{s}}(A, C), \delta_{p_{s}}(B, C)\right\}$.

Let $\left(X, p_{s}\right)$ be a partial symmetric spaces. We define

$$
H_{p_{s}}(A, B)=\max \left\{\delta p_{s}(A, B), \delta p_{s}(B, A)\right\} .
$$


Proposition 2.2. [19] Let $\left(X, p_{s}\right)$ be a complete partial symmetric space, for $A, B, C, D \in C B^{p_{s}}(X)$, the following condition holds:

(1) $H_{p_{s}}(A, A) \leq H_{p_{s}}(A, B)$;

(2) $H_{p_{s}}(A, B)=H_{p_{s}}(B, A)$;

(3) $H_{p_{s}}(A \cup B, C \cup D)=\max \left\{H_{p_{s}}(A, C), H_{p_{s}}(B, D)\right\}$.

Proposition 2.3. [19] Let $\left(X, p_{s}\right)$ be a complete partial symmetric space, for $A, B \in C B^{p_{s}}(X)$, we have

$$
H_{p_{s}}(A, B)=0 \Rightarrow A=B .
$$

Lemma 2.2. [19] Let $\left(X, p_{s}\right)$ be a partial symmetric space for $A, B \in C B^{p_{s}}(X)$. Then, for any $h<1$ and $a \in A$, there exists $b \in B$ such that

$$
p_{s}(a, b)=h H_{p_{s}}(A, B) .
$$

Definition 2.5. [2] A map $T: X \longrightarrow C B(X)$ is said to be multi-valued contraction if there exist $\lambda \in[0,1)$ such that

$$
H(T x, T y) \leq \lambda d(x, y),
$$

for all $x, y \in X$.

Assad and Kirk in [3], introduced the following lemma in convex metric space.

Lemma 2.3. [3] If $C$ is a nonempty closed subset of a metrically convex metric space $(X, d)$, then for any $x \in C$, $y \notin C$, there exists a point $z \in \partial C$ (the boundary of $C$ ) such that

$$
d(x, z)+d(z, y)=d(x, y) .
$$

Kumar and Rugumisa formulated the following lemma:

Lemma 2.4. [24] Let $C$ be a non-empty subset of a metrically convex partial metric space $(X, p)$ which is closed in $\left(X, p^{s}\right)$. If $x \in C$ and $y \in X \notin C$, then there exists a point $z \in \partial C$ (the boundary of $C$ ) such that

$$
p(x, z)+p(z, y)=p(x, y)+p(z, z) .
$$

Assad and Kirk [3] proved the following results in metrically convex metric spaces

Theorem 2.2. [3] Let $(X, d)$ be a complete and metrically convex metric space, $C$ a non empty closed subset of $X$ and $T: C \longrightarrow C B(X)$ be a mapping. Assume that the following conditions are holds:

(i) $T x \in C$ for each $x \in \partial C$,

(ii) there exists $k \in(0,1), \forall x, y \in C$,

$$
H(T x, T y) \leq k d(x, y)
$$

Then $T$ has a unique fixed point in $C$.

We shall use the following lemma, which is given by Kumar and Rugumisa [24] in metrically convex partial space and will be helpful in the proof of our main results.

Lemma 2.5. [24] If $C$ is a nonempty closed subset of a metrically convex partial symmetric space $\left(X, p_{s}\right)$, then for any $x \in C, y \notin C$, there exists a point $z \in \partial C$ (the boundary of $C$ ) such that

$$
p_{s}(x, z)+p_{s}(z, y)=p_{s}(x, y)+p_{s}(z, z) .
$$




\section{Main Results}

In this section, we prove our main theorem as given below:

Theorem 3.1. Let $\left(X, p_{S}\right)$ be a complete metrically convex partial symmetric spaces, $C$ a non empty closed subset of $X$ and $T: C \longrightarrow C B^{p_{s}}(X)$ be a multivalued mapping. Assume that the following conditions are holds:

(i) $T x \in C$ for each $x \in \partial C$,

(ii) $T$ is continuous,

(iii) There exists $k \in(0,1), \forall x, y \in C$ with $H_{p_{s}}(T x, T y)>0$; such that

$$
\begin{aligned}
H_{p_{s}}(T x, T y) \leq & k \max \left\{p_{s}(x, y), p_{s}(x, T x), p_{s}(y, T y),\right. \\
& \left.\frac{p_{s}(x, T y)+p_{s}(y, T x)}{2}\right\} .
\end{aligned}
$$

Then, $T$ has a unique fixed point, if there exists $x \in X$ such that $p_{S}(x, x)=0$.

Proof. Suppose that $T$ has no fixed points then $p_{s}(x, T x)>0$ for all $x \in C$. We start by constructing two sequences $\left\{x_{n}\right\}$ and $\left\{y_{n}\right\}$, respectively in the following way.

Let $x_{0} \in \partial C$ and $y_{1} \in T x_{0}$, if $y_{1} \in C$, let $x_{1}=y_{1}$, then our proof would be completed. Since $T$ is a multivalued mapping $x_{1} \in T x_{0}$. If $y_{1} \notin C$, then, by Lemma 2.5 there exists $x_{1} \in \partial C$ such that

$$
p_{s}\left(x_{0}, x_{1}\right)+p_{s}\left(x_{1}, y_{1}\right)=p_{s}\left(x_{0}, y_{1}\right)+p_{s}\left(x_{1}, x_{1}\right)
$$

Thus for $x_{1} \in C$ and using Lemma 2.2, we can choose $y_{2} \in T x_{1}$ such that

$$
p_{s}\left(y_{1}, y_{2}\right) \leq h H_{p_{s}}\left(T x_{0}, T x_{1}\right)
$$

For $h=\frac{1}{\sqrt{k}}$, Equation (10) becomes

$$
p_{s}\left(y_{1}, y_{2}\right) \leq \frac{1}{\sqrt{k}} H_{p_{s}}\left(T x_{0}, T x_{1}\right)
$$

However, if $y_{2} \in C$, let $x_{2}=y_{2}$. If $y_{2} \notin C$. Then, using Lemma 2.5 there exists $x_{2} \in \partial C$ such that

$$
p_{s}\left(x_{1}, x_{2}\right)+p_{s}\left(x_{2}, y_{2}\right)=p_{s}\left(x_{1}, y_{2}\right)+p_{s}\left(x_{2}, x_{2}\right) \text {. }
$$

Thus $x_{2} \in C$ and using Lemma 2.2, we can choose $y_{3} \in T x_{2}$ such that

$$
p_{s}\left(y_{2}, y_{3}\right) \leq h H_{p_{s}}\left(T x_{1}, T x_{2}\right) \text {. }
$$

Again for $h=\frac{1}{\sqrt{k}}$ in (11) we have

$$
p_{s}\left(y_{2}, y_{3}\right) \leq \frac{1}{\sqrt{k}} H_{p_{s}}\left(T x_{1}, T x_{2}\right)
$$

Continuing the arguments and following the iterative process, we obtain two sequences $\left\{x_{n}\right\}$ and $\left\{y_{n}\right\}$, such that for $n \in \mathbb{N}$, we have

(i) $y_{n+1} \in T x_{n}$;

(ii) $p_{s}\left(y_{n}, y_{n+1}\right) \leq\left(\frac{1}{\sqrt{k}}\right) H_{p_{s}}\left(T x_{n-1}, T x_{n}\right)$;

(iii) $y_{n} \in C \Rightarrow y_{n}=x_{n}$;

(iv) $y_{n} \neq x_{n}$, whenever $y_{n} \notin C$ and $x_{n} \in \partial C$ implies that

$$
p_{s}\left(x_{n-1}, x_{n}\right)+p_{s}\left(x_{n}, y_{n}\right)=p_{s}\left(x_{n-1}, y_{n}\right)+p_{s}\left(x_{n}, x_{n}\right) \text {. }
$$

Now, we claim that $\left\{x_{n}\right\}$ is a Cauchy sequence. Assume that $P$ and $Q$ are two sets in $C$ such that

$$
\begin{aligned}
& P=\left\{x_{i} \in\left\{x_{n}\right\}: x_{i}=y_{i}, i=1,2, \ldots\right\}, \\
& Q=\left\{x_{i} \in\left\{x_{n}\right\}: x_{i} \neq y_{i}, i=1,2, \ldots\right\} .
\end{aligned}
$$


Obviously, if $x_{n} \in Q$, for some $n$, then $x_{n}$ and $x_{n+1}$ belong to $P$;

Thus, we have three possible cases.

\section{Case I}

If $x_{n}, x_{n+1} \in P$, then $y_{n}=x_{n}, y_{n+1}=x_{n+1}$. Assume that $x_{n}=y_{n}=T x_{n-1}$

$x_{n+1}=y_{n+1}=T x_{n}$. Then, we have

$$
p_{s}\left(x_{n}, x_{n+1}\right)=p_{s}\left(y_{n}, y_{n+1}\right)=p_{s}\left(T x_{n-1}, T x_{n}\right) .
$$

Since $\left\{x_{n}\right\} \in C$ for all $n \in \mathbb{N}$, by using (8), we shows that $x_{n}, x_{n+1} \in \partial C$.

Now,

$$
\begin{gathered}
p_{s}\left(x_{n}, x_{n+1}\right)=p_{s}\left(T x_{n-1}, T x_{n}\right) . \\
H_{p_{s}}\left(T x_{n-1}, T x_{n}\right) \leq \quad k \max \left\{p_{s}(x, y), p_{s}(x, T x), p_{s}(y, T y),\right. \\
\left.\frac{p_{s}(x, T y)+p_{s}(y, T x)}{2}\right\}
\end{gathered}
$$

Thus, letting $x=x_{n-1}$ and $y=x_{n}$ in (12), we have

$$
\begin{aligned}
H_{p_{s}}\left(T x_{n-1}, T x_{n}\right) \leq & k \max \left\{p_{s}\left(x_{n-1}, x_{n}\right), p_{s}\left(x_{n-1}, T x_{n-1}\right), p_{s}\left(x_{n}, T x_{n}\right),\right. \\
& \left.\frac{p_{s}\left(x_{n-1}, T x_{n}\right)+p_{s}\left(x_{n}, T x_{n-1}\right)}{2}\right\} \\
\leq & k \max \left\{p_{s}\left(x_{n-1}, x_{n}\right), p_{s}\left(x_{n-1}, x_{n}\right), p_{s}\left(x_{n}, x_{n+1}\right),\right. \\
& \left.\frac{p_{s}\left(x_{n-1}, x_{n+1}\right)+p_{s}\left(x_{n}, x_{n}\right)}{2}\right\} .
\end{aligned}
$$

By Lemma 2.5

$$
p_{s}\left(x_{n-1}, x_{n}\right)+p_{s}\left(x_{n}, x_{n+1}\right)=p_{s}\left(x_{n-1}, x_{n+1}\right)+p_{s}\left(x_{n}, x_{n}\right) \text {. }
$$

Using (14) in (13), we obtain

$$
\begin{aligned}
H_{p_{s}}\left(T x_{n-1}, T x_{n}\right) \leq & k \max \left\{p_{s}\left(x_{n-1}, x_{n}\right), p_{s}\left(x_{n-1}, x_{n}\right), p_{s}\left(x_{n}, x_{n+1}\right),\right. \\
& \left.\frac{p_{s}\left(x_{n-1}, x_{n}\right)+p_{s}\left(x_{n}, x_{n+1}\right)-p_{s}\left(x_{n}, x_{n}\right)+p_{s}\left(x_{n}, x_{n}\right)}{2}\right\} \\
\leq & \left.k \max \left\{p_{s}\left(x_{n-1}, x_{n}\right), p_{s}\left(x_{n}, x_{n+1}\right), \frac{p_{s}\left(x_{n-1}, x_{n}\right)+p_{s}\left(x_{n}, x_{n+1}\right)}{2}\right)\right\} \\
\leq & k \max \left\{p_{s}\left(x_{n-1}, x_{n}\right), p_{s}\left(x_{n}, x_{n+1}\right\}\right.
\end{aligned}
$$

By using Lemma 2.2 and taking $h=\frac{1}{\sqrt{k}}$, we have

$$
\begin{aligned}
p_{s}(x, y) & =h H_{p_{s}}(T x, T y), \\
& =\frac{1}{\sqrt{k}} H_{p_{s}}\left(T x_{n-1}, T x_{n}\right), \\
p_{s}\left(x_{n}, x_{n+1}\right) & \leq(\sqrt{k})^{n} \max \left(p_{s}\left(x_{n-1}, x_{n}\right), p_{s}\left(x_{n}, x_{n+1}\right)\right) .
\end{aligned}
$$

Which shows that, either

$$
p_{s}\left(x_{n}, x_{n+1}\right) \leq(\sqrt{k})^{n} p_{s}\left(x_{n-1}, x_{n}\right) \text {, }
$$

or

$$
p_{s}\left(x_{n}, x_{n+1}\right) \leq(\sqrt{k})^{n} p_{s}\left(x_{n}, x_{n+1}\right) \text {, }
$$


is the maximum of (15). Inductively, using (16) we proceed as follows:

Let $x_{n} \in T x_{n}$ and $p_{s}\left(x_{n}, x_{n+1}\right) \leq(\sqrt{k})^{n} p_{s}\left(x_{n-1}, x_{n}\right)$. For all $n \geq 1$ and $n, m>\mathbb{N}$, we have

$$
\begin{gathered}
p_{s}\left(x_{n}, x_{m}\right) \leq p_{s}\left(x_{n}, x_{n+1}\right)+p_{s}\left(x_{n+1}, x_{n+2}\right)+\cdots+p_{s}\left(x_{m-1}, x_{m+n}\right) . \\
p_{s}\left(x_{n}, x_{m}\right) \leq\left\{(\sqrt{k})^{n}+(\sqrt{k})^{n+1}+\cdots+(\sqrt{k})^{n+m-1}\right\} p_{s}\left(x_{0}, x_{1}\right) .
\end{gathered}
$$

Applying the sum of G.P, we obtain

$$
\begin{gathered}
p_{s}\left(x_{n}, x_{m}\right) \leq \frac{(\sqrt{k})^{n}}{1-\sqrt{k}} p_{s}\left(x_{0}, x_{1}\right) . \\
\lim _{n, m \rightarrow \infty} p_{s}\left(x_{n}, x_{m}\right)=0 .
\end{gathered}
$$

Similarly, using Equation (17). Assume that

$$
(\sqrt{k})^{n} \max \left(p_{s}\left(x_{n-1}, x_{n}\right), p_{s}\left(x_{n}, x_{n+1}\right)\right)=(\sqrt{k})^{n} p_{s}\left(x_{n}, x_{n+1}\right) .
$$

So, we have

$$
p_{s}\left(x_{n}, x_{n+1}\right) \leq(\sqrt{k})^{n} p_{s}\left(x_{n}, x_{n+1}\right) .
$$

For continuity of $T$ in the above inequality, we obtain

$$
p_{s}\left(x_{n}, x_{n+1}\right) \leq(\sqrt{k})^{n} p_{s}\left(x_{n}, x_{n+1}\right) \leq p_{s}\left(x_{n}, x_{n+1}\right),
$$

which is a contradiction and so

$$
p_{s}\left(x_{n}, x_{n+1}\right) \leq(\sqrt{k})^{n} p_{s}\left(x_{n-1}, x_{n}\right) .
$$

Cosequently, for $y_{n}, y_{n+1} \in P$ we have

$$
p_{s}\left(y_{n}, y_{n+1}\right)=p_{s}\left(x_{n}, x_{n+1}\right)=p_{s}\left(T y_{n-1}, T y_{n}\right) .
$$

Since $\left\{y_{n}\right\} \in C$ for all $n \in \mathbb{N}$, by using (8), we shows that $y_{n}, y_{n+1} \in \partial C$.

Now,

$$
\begin{gathered}
p_{s}\left(y_{n}, y_{n+1}\right)=p_{s}\left(T y_{n-1}, T y_{n}\right) . \\
H_{p_{s}}\left(T y_{n-1}, T y_{n}\right) \leq \quad k \max \left\{p_{s}(x, y), p_{s}(x, T x), p_{s}(y, T y),\right. \\
\left.\frac{p_{s}(x, T y)+p_{s}(y, T x)}{2}\right\}
\end{gathered}
$$

Thus, letting $x=y_{n-1}$ and $y=y_{n}$ in (20), we have

$$
\begin{aligned}
H_{p_{s}}\left(T y_{n-1}, T y_{n}\right) \leq & k \max \left\{p_{s}\left(y_{n-1}, y_{n}\right), p_{s}\left(y_{n-1}, T y_{n-1}\right), p_{s}\left(y_{n}, T y_{n}\right),\right. \\
& \left.\frac{p_{s}\left(y_{n-1}, T y_{n}\right)+p_{s}\left(y_{n}, T y_{n-1}\right)}{2}\right\} \\
\leq & k \max \left\{p_{s}\left(y_{n-1}, y_{n}\right), p_{s}\left(y_{n-1}, y_{n}\right), p_{s}\left(y_{n}, y_{n+1}\right),\right. \\
& \left.\frac{p_{s}\left(y_{n-1}, y_{n+1}\right)+p_{s}\left(y_{n}, y_{n}\right)}{2}\right\} .
\end{aligned}
$$

By Lemma 2.5

$$
p_{s}\left(y_{n-1}, y_{n}\right)+p_{s}\left(y_{n}, y_{n+1}\right)=p_{s}\left(y_{n-1}, y_{n+1}\right)+p_{s}\left(y_{n}, x_{n}\right) \text {. }
$$


Applying (22) in (21), one obtains

$$
\begin{aligned}
H_{p_{s}}\left(T y_{n-1}, T y_{n}\right) \leq & k \max \left\{p_{s}\left(y_{n-1}, y_{n}\right), p_{s}\left(y_{n-1}, y_{n}\right), p_{s}\left(y_{n}, y_{n+1}\right),\right. \\
& \left.\frac{p_{s}\left(y_{n-1}, y_{n}\right)+p_{s}\left(y_{n}, y_{n+1}\right)-p_{s}\left(y_{n}, y_{n}\right)+p_{s}\left(y_{n}, y_{n}\right)}{2}\right\} \\
\leq & \left.k \max \left\{p_{s}\left(y_{n-1}, y_{n}\right), p_{s}\left(y_{n}, y_{n+1}\right), \frac{p_{s}\left(y_{n-1}, y_{n}\right)+p_{s}\left(y_{n}, y_{n+1}\right)}{2}\right)\right\} \\
\leq & k \max \left\{p_{s}\left(y_{n-1}, y_{n}\right), p_{s}\left(y_{n}, x_{n+1}\right\}\right.
\end{aligned}
$$

By using Lemma 2.2 and taking $h=\frac{1}{\sqrt{k}}$, we have

$$
\begin{aligned}
p_{s}(x, y) & =h H_{p_{s}}(T x, T y), \\
& =\frac{1}{\sqrt{k}} H_{p_{s}}\left(T y_{n-1}, T y_{n}\right), \\
p_{s}\left(y_{n}, y_{n+1}\right) & \leq(\sqrt{k})^{n} \max \left(p_{s}\left(y_{n-1}, y_{n}\right), p_{s}\left(y_{n}, y_{n+1}\right)\right) .
\end{aligned}
$$

Which shows that, either

$$
p_{s}\left(y_{n}, y_{n+1}\right) \leq(\sqrt{k})^{n} p_{s}\left(y_{n-1}, y_{n}\right)
$$

or

$$
p_{s}\left(y_{n}, y_{n+1}\right) \leq(\sqrt{k})^{n} p_{s}\left(y_{n}, y_{n+1}\right),
$$

is the maximum of (23). By following similar steps from above, we reach the same results as in equation (19).

\section{Case II}

If $x_{n} \in P, x_{n+1} \in Q$. Assume that $x_{n}=y_{n}=T x_{n-1}$ and $x_{n+1} \neq y_{n+1}=T x_{n}$. Then, we have

$$
\begin{aligned}
p_{s}\left(x_{n}, x_{n+1}\right) & \leq p_{s}\left(x_{n}, y_{n+1}\right), \\
& =p_{s}\left(y_{n}, y_{n+1}\right), .
\end{aligned}
$$

Since $\left\{x_{n}\right\} \in C$ for all $n \in \mathbb{N}$, using contraction condition we shows that $x_{n}, x_{n-1} \in \partial C$.

Now,

$$
p_{s}\left(x_{n}, x_{n+1}\right) \leq h H p_{s}\left(T x_{n-1}, T x_{n}\right) .
$$

By using $x=x_{n-1}$ and $y=x_{n}$ in (8), we have

$$
\begin{aligned}
H_{p_{s}}\left(T x_{n-1}, T x_{n}\right) \leq & k \max \left\{p_{s}\left(x_{n-1}, x_{n}\right), p_{s}\left(x_{n-1}, T x_{n-1}\right), p_{s}\left(x_{n}, T x_{n}\right),\right. \\
& \left.\frac{p_{s}\left(x_{n-1}, T x_{n}\right)+p_{s}\left(x_{n}, T x_{n-1}\right)}{2}\right\}, \\
\leq & k \max \left\{p_{s}\left(x_{n-1}, x_{n}\right), p_{s}\left(x_{n-1}, x_{n}\right), p_{s}\left(x_{n}, x_{n+1}\right),\right. \\
& \left.\frac{p_{s}\left(x_{n-1}, x_{n+1}\right)+p_{s}\left(x_{n}, x_{n}\right)}{2}\right\} .
\end{aligned}
$$

By Lemma 2.5, we have

$$
p_{s}\left(x_{n-1}, x_{n}\right)+p_{s}\left(x_{n}, x_{n+1}\right)=p_{s}\left(x_{n-1}, x_{n+1}\right)+p_{s}\left(x_{n}, x_{n}\right) .
$$

Apply Equation (27) in (26), we obtain

$$
\begin{aligned}
H_{p_{s}}\left(T x_{n-1}, T x_{n}\right) \leq & k \max \left\{p_{s}\left(x_{n-1}, x_{n}\right), p_{s}\left(x_{n-1}, x_{n}\right), p_{s}\left(x_{n}, x_{n+1}\right),\right. \\
& \left.\frac{p_{s}\left(x_{n-1}, x_{n}\right)+p_{s}\left(x_{n}, x_{n+1}\right)-p_{s}\left(x_{n}, x_{n}\right)+p_{s}\left(x_{n}, x_{n}\right)}{2}\right\} \\
\leq & k \max \left\{p_{s}\left(x_{n-1}, x_{n}\right), p_{s}\left(x_{n}, x_{n+1}\right),\right. \\
& \left.\frac{p_{s}\left(x_{n-1}, x_{n}\right)+p_{s}\left(x_{n}, x_{n+1}\right)}{2}\right\} \\
\leq & k \max \left\{p_{s}\left(x_{n-1}, x_{n}\right), p_{s}\left(x_{n}, x_{n+1}\right)\right\}
\end{aligned}
$$


By using Lemma 2.2 and taking $h=\frac{1}{\sqrt{k}}$, we have

$$
\begin{aligned}
p_{s}(x, y) & =h H_{p_{s}}(T x, T y), \\
& =\frac{1}{\sqrt{k}} H_{p_{s}}\left(T x_{n-1}, T x_{n}\right), \\
p_{s}\left(x_{n}, x_{n+1}\right) & \leq(\sqrt{k})^{n} \max \left\{p_{s}\left(x_{n-1}, x_{n}\right), p_{s}\left(x_{n}, x_{n+1}\right)\right\} .
\end{aligned}
$$

Thus

$$
p_{s}\left(x_{n}, x_{n+1}\right) \leq(\sqrt{k})^{n} p_{s}\left(x_{n-1}, x_{n}\right) .
$$

We have the same result as in the case I.

\section{Case III}

If $x_{n} \in Q, x_{n+1} \in P, y_{n} \neq x_{n}, x_{n-1} \in P, x_{n+1} \in P$. Assume that $x_{n+1}=T x_{n}, x_{n} \neq y_{n}=T x_{n-1}$. Then, we have

$$
\begin{aligned}
& p_{s}\left(x_{n}, x_{n+1}\right) \leq p_{s}\left(x_{n}, y_{n}\right)+p_{s}\left(y_{n}, x_{n+1}\right), \\
& p_{s}\left(x_{n}, x_{n+1}\right) \leq p_{s}\left(x_{n}, y_{n}\right)+p_{s}\left(y_{n}, y_{n+1}\right)
\end{aligned}
$$

From Case I, if $y_{n}, y_{n+1} \in P \times Q$, then (29) becomes

$$
\begin{aligned}
& p_{s}\left(x_{n}, x_{n+1}\right) \leq p_{s}\left(x_{n}, y_{n}\right)+H_{p_{s}}\left(T x_{n-1}, T x_{n}\right), \\
& p_{s}\left(x_{n}, x_{n+1}\right) \leq p_{s}\left(x_{n}, y_{n}\right)+(\sqrt{k})^{n}\left(p_{s}\left(x_{n-1}, x_{n}\right) .\right.
\end{aligned}
$$

Using (iv) we have

$$
p_{s}\left(x_{n-1}, x_{n}\right)+p_{s}\left(x_{n}, y_{n}\right)=p_{s}\left(x_{n-1}, y_{n}\right)+p_{s}\left(x_{n}, x_{n}\right) .
$$

Since, $p_{s}\left(x_{n}, x_{n}\right) \Rightarrow p_{s}(x, x)=0$, the above equality becomes

$$
\begin{aligned}
p_{s}\left(x_{n-1}, x_{n}\right)+p_{s}\left(x_{n}, y_{n}\right) & =p_{s}\left(x_{n-1}, y_{n}\right), \\
p_{s}\left(x_{n}, y_{n}\right) & =p_{s}\left(x_{n-1}, y_{n}\right)-p_{s}\left(x_{n-1}, x_{n}\right) .
\end{aligned}
$$

Applying (31) in (30), we get

$$
p_{s}\left(x_{n}, x_{n+1}\right) \leq p_{s}\left(x_{n-1}, y_{n}\right)-p_{s}\left(x_{n-1}, x_{n}\right)+(\sqrt{k})^{n}\left(p_{s}\left(x_{n-1}, x_{n}\right) .\right.
$$

Since, $k<1$ we obtain

$$
\begin{gathered}
p_{s}\left(x_{n}, x_{n+1}\right) \leq p_{s}\left(x_{n-1}, y_{n}\right), \\
\leq p_{s}\left(y_{n-1}, y_{n}\right) \\
p_{s}\left(y_{n-1}, y_{n}\right)=p_{s}\left(y_{n-1}, T x_{n-1}\right) \leq h H_{p s}\left(T x_{n-2}, T x_{n-1}\right),
\end{gathered}
$$

Since, $x_{n} \in Q \Rightarrow x_{n-1} \in P$. Thus,

$$
p_{s}\left(x_{n}, x_{n+1}\right)=h H_{p s}\left(T x_{n-2}, T x_{n-1}\right) .
$$

Since $x_{n-1} \in C$ for all $n \in \mathbb{N}$, by using (8) we can show that $x_{n-2}, x_{n-1} \in \partial C$.

By substituting $x=x_{n-2}$ and $y=x_{n-1}$ in (8), we have

$$
\begin{aligned}
H_{p_{s}}\left(T x_{n-2}, T x_{n-1}\right) \leq & k \max \left\{p_{s}\left(x_{n-2}, x_{n-1}\right), p_{s}\left(x_{n-2}, T x_{n-2}\right), p_{s}\left(x_{n-1}, T x_{n-1}\right),\right. \\
& \left.\frac{p_{s}\left(x_{n-2}, T x_{n-1}\right)+p_{s}\left(x_{n-1}, T x_{n-2}\right)}{2}\right\} . \\
\leq & k \max \left\{p_{s}\left(x_{n-2}, x_{n-1}\right), p_{s}\left(x_{n-2}, x_{n-1}\right), p_{s}\left(x_{n-1}, x_{n}\right),\right. \\
& \left.\frac{p_{s}\left(x_{n-2}, x_{n}\right)+p_{s}\left(x_{n-1}, x_{n-1}\right)}{2}\right\} .
\end{aligned}
$$


By using Lemma 2.5, we have

$$
\begin{aligned}
\leq & k \max \left\{p_{s}\left(x_{n-2}, x_{n-1}\right), p_{s}\left(x_{n-2}, x_{n-1}\right), p_{s}\left(x_{n-1}, x_{n}\right),\right. \\
& \left.\frac{p_{s}\left(x_{n-2}, x_{n-1}\right)+p_{s}\left(x_{n-1}, x_{n}\right)-p_{s}\left(x_{n-1}, x_{n-1}\right)+p_{s}\left(x_{n-1}, x_{n-1}\right)}{2}\right\} \\
\leq & k \max \left\{p_{s}\left(x_{n-2}, x_{n-1}\right), p_{s}\left(x_{n-2}, x_{n-1}\right), p_{s}\left(x_{n-1}, x_{n}\right),\right. \\
& \left.\frac{p_{s}\left(x_{n-2}, x_{n-1}\right)+p_{s}\left(x_{n-1}, x_{n}\right)}{2}\right\} \\
\leq & k \max \left\{p_{s}\left(x_{n-2}, x_{n-1}\right), p_{s}\left(x_{n-1}, x_{n}\right)\right\}
\end{aligned}
$$

Using Lemma 2.2 and taking $h=\frac{1}{\sqrt{k}}$ in (33), we get

$$
\begin{aligned}
p_{s}(x, y) & =h H_{p_{s}}(T x, T y), \\
p_{s}\left(x_{n}, x_{n+1}\right) & \leq \frac{1}{\sqrt{k}} H_{p_{s}}\left(T x_{n-2}, T x_{n-1}\right), \\
& \leq \sqrt{k} \max \left\{p_{s}\left(x_{n-2}, x_{n-1}\right), p_{s}\left(x_{n-1}, x_{n}\right)\right\},
\end{aligned}
$$

which implies that, either

$$
p_{s}\left(x_{n}, x_{n+1}\right) \leq \sqrt{k} \max \left\{p_{s}\left(x_{n-1}, x_{n}\right)\right\} .
$$

or

$$
p_{s}\left(x_{n}, x_{n+1}\right) \leq \sqrt{k} \max \left\{p_{s}\left(x_{n-2}, x_{n-1}\right)\right\}
$$

Thus, for $n \geq 2$, we have

$$
p_{s}\left(x_{n}, x_{n+1}\right)=\left\{\begin{array}{l}
(\sqrt{k})^{n} p_{s}\left(x_{n-2}, x_{n-1}\right) \\
(\sqrt{k})^{n} p_{s}\left(x_{n-1}, x_{n}\right)
\end{array}\right.
$$

Letting,

$$
\delta=\max \left(p_{s}\left(x_{n-2}, x_{n-1}\right), p_{s}\left(x_{n-1}, x_{n}\right)\right) .
$$

Thus in all three cases, Case I, Case II, Case III, we have

$$
\begin{aligned}
p_{s}\left(x_{n}, x_{n+1}\right) & \leq(\sqrt{k})^{n} \max \left\{p_{s}\left(x_{n-2}, x_{n-1}\right), p_{s}\left(x_{n-1}, x_{n}\right)\right\} . \\
& \leq(\sqrt{k})^{n} \delta .
\end{aligned}
$$

Inductively for all cases, there exists a sequence $P_{n} \in C$ such that

$$
x_{n+1} \in T x_{n} \text { and } p_{s}\left(x_{n}, x_{n+1}\right) \leq(\sqrt{k})^{n} \max \left(p_{s}\left(x_{0}, x_{1}\right), p_{s}\left(x_{1}, x_{2}\right)\right) .
$$

For $n>1$, since $n, m \in \mathbb{N}, n>m$, we obtain

$$
\begin{aligned}
p_{s}\left(x_{n}, x_{m}\right) & \leq \sum_{i=n}^{m-1} p_{s}\left(x_{i}, x_{i+1}\right) \\
& \leq \sum_{i=n}^{m-1}(\sqrt{k})^{i} \delta \\
& \leq \sum_{i=n}^{\infty}(\sqrt{k})^{i} \delta \\
& \leq \delta \frac{(\sqrt{k})^{n}}{1-\sqrt{k}} .
\end{aligned}
$$

As $n, m \rightarrow \infty$, we obtain

$$
\lim _{m, n \rightarrow \infty} p_{s}\left(x_{n}, x_{m}\right)=0 .
$$


Therefore, $\left\{x_{n}\right\}$ is a $p_{s}$ - Cauchy sequence in $C$. There exists $x \in C$ such that $\left\{x_{n}\right\}$ is a $p_{s}$ - converges to $x$.

$$
\begin{aligned}
\lim _{n, m \rightarrow \infty} p_{s}\left(x_{n}, x_{m}\right) & =\lim _{n \rightarrow \infty} p_{s}\left(x_{n}, x\right), \\
& =p_{s}(x, x)=0 .
\end{aligned}
$$

We proved that $\left\{x_{n}\right\}$ is Cauchy sequence. Also, since $\left(X, p_{s}\right)$ is a complete convex partial symmetric space, there is $x \in X$ such that $x_{n} \rightarrow x$, and $p(x, x)=0$.

Now we will show that $x$ is a fixed point of $T$. We do this by showing that $p_{s}(x, T x)=p_{s}(x, x)=0$. Suppose $p_{s}(x, T x)>0$. Then there exists some $n_{0} \in \mathbb{N}$ such that $p_{s}\left(x_{n}, T x\right)>0$ for all $n>n_{0}$.

By substituting $x=x_{n}$ and $y=x$ in (8), we have

$$
\begin{aligned}
H_{p_{s}}\left(T x_{n}, T x\right) \leq & k \max \left\{p_{s}\left(x_{n}, x\right) p_{s}\left(x_{n}, T x_{n}\right), p_{s}(x, T x),\right. \\
& \left.\frac{p_{s}\left(x_{n}, T x\right)+p_{s}\left(x, T x_{n}\right)}{2}\right\} .
\end{aligned}
$$

Taking $n \rightarrow \infty$ in (35) and applying the fact that $T$ is continuous, we get

$$
\begin{aligned}
& H_{p_{s}}\left(T x_{n}, T x\right) \leq k \max \left\{p_{s}(x, x), p_{s}(x, T x), p_{s}(x, T x), \frac{p_{s}(x, T x)+p_{s}(x, T x)}{2}\right\} . \\
& H_{p_{s}}\left(T x_{n}, T x\right) \leq k p_{s}(x, T x)
\end{aligned}
$$

By Lemma 2.2 implies that

$$
p_{s}\left(x_{n}, x\right)=\frac{1}{\sqrt{k}} H_{p_{s}}\left(T x_{n}, T x\right)
$$

Using (36) and $n \rightarrow \infty$ in above inequality we obtain

$$
p_{s}(x, x) \leq \frac{1}{\sqrt{k}} k p_{s}(x, T x) .
$$

By the continuity of $T$, we get

$$
0 \leq p_{s}(x, T x)
$$

Therefore,

$$
p_{s}(x, x)=p_{s}(x, T \chi)=0,
$$

which is a contradiction. Hence, $x$ is a fixed point of $T$.

Next, we claim that $x$ is unique. Suppose that $x^{\star}$ is another element in $C$ such that $T x^{\star}=x^{\star}, T x=x$. Then

$$
\begin{aligned}
& H_{p_{s}}\left(T x, T x^{\star}\right) \leq k \max \left\{p_{s}\left(x, x^{\star}\right), p_{s}(x, T x), p_{s}\left(x^{\star}, T x^{\star}\right), \frac{p_{s}\left(x, T x^{\star}\right)+p_{s}\left(x^{\star}, T x\right)}{2}\right\} . \\
& H_{p_{s}}\left(T x, T x^{\star}\right) \leq k p_{s}\left(x, x^{\star}\right)
\end{aligned}
$$

By Lemma 2.2 implies that

$$
p_{s}\left(x, x^{\star}\right)=\frac{1}{\sqrt{k}} H_{p_{s}}\left(T x, T x^{\star}\right) .
$$

Using (37) and in above inequality we obtain

$$
p_{s}\left(x, x^{\star}\right) \leq \frac{1}{\sqrt{k}} k p_{s}\left(x, x^{\star}\right) .
$$

By the continuity of $T$, we get

$$
0 \leq p_{s}\left(x, x^{\star}\right)
$$

Hence

$$
p_{s}\left(x, x^{\star}\right)=0 \text {, }
$$

implying that $x=x^{\star}$. Which is a contradiction. Hence, the fixed point is not unique. 
We give the following corollary, which is motivated from Ali et al. [16].

Corollary 3.1. Let $\left(X, p_{s}\right)$ be a complete metrically convex partial symmetric space, suppose $D$ is a nonempty subset of $X$. Let $T: D \rightarrow C B^{p_{s}}(X)$ be a mapping such that $T x \neq 0$ for each $x, y \in D, q, r \geq 2$ with $H_{p_{s}}(T x, T y) \geq$ 0 , we have

$$
H_{p_{s}}(T x, T y)=\psi(M(x, y))
$$

where

$$
M(x, y)=\max \left\{p(x, y), \frac{p(x, T x)+p(y, T y)}{q}, \frac{p(x, T y)+p(y, T x)}{r}\right\},
$$

and $\psi$ is an increasing function in $\Psi$. Also assume that the following conditions hold:

(i) there exist $x_{0} \in \partial D$ and $x_{1} \in T x_{0}$ such that $T x_{0} \in D$,

(ii) $T$ is continuous,

(iiii)for any sequence $\left\{x_{n}\right\}$ in $D$ such that $x_{n} \rightarrow x$ as $n \rightarrow \infty$ and $x_{n} \preceq x_{n+1}$ for each $n \in \mathbb{N} \cup\{0\}, p_{s}\left(x_{n}, x\right) \rightarrow$ $p_{s}(x, x)$ as $n \rightarrow \infty$.

Then $T$ has a fixed point.

Proof. Suppose that $T$ has no fixed points then $p_{s}\left(x_{0}, T x_{0}\right)>0$ for all $x \in D$. We start by constructing two sequences $\left\{x_{n}\right\}$ and $\left\{y_{n}\right\}$, respectively, in the following way.

Let $x_{0} \in \partial D$ and $y_{1} \in T x_{0}$, if $y_{1} \in D$, let $x_{1}=y_{1}$, then our proof would be completed. Since $T$ is a multivalued mapping $x_{1} \in T x_{0}$. If $y_{1} \notin D$, then, by Lemma 2.5 there exists $x_{1} \in \partial D$ such that

$$
p_{s}\left(x_{0}, x_{1}\right)+p_{s}\left(x_{1}, y_{1}\right)=p_{s}\left(x_{0}, y_{1}\right)+p_{s}\left(x_{1}, x_{1}\right) \text {. }
$$

Thus for $x_{1} \in D$ and using Lemma 2.2, we can choose $y_{2} \in T x_{1}$ such that

$$
p_{s}\left(y_{1}, y_{2}\right) \leq h H_{p_{s}}\left(T x_{0}, T x_{1}\right) \text {. }
$$

For $h=\frac{1}{\sqrt{k}}$, equation (39) becomes

$$
p_{s}\left(y_{1}, y_{2}\right) \leq \frac{1}{\sqrt{k}} H_{p_{s}}\left(T x_{0}, T x_{1}\right) .
$$

However, if $y_{2} \in D$, let $x_{2}=y_{2}$. If $y_{2} \notin D$. Then, using Lemma 2.5 there exists $x_{2} \in \partial D$ such that

$$
p_{s}\left(x_{1}, x_{2}\right)+p_{s}\left(x_{2}, y_{2}\right)=p_{s}\left(x_{1}, y_{2}\right)+p_{s}\left(x_{2}, x_{2}\right) \text {. }
$$

Thus $x_{2} \in D$ and using Lemma 2.2, we can choose $y_{3} \in T x_{2}$ such that

$$
p_{s}\left(y_{2}, y_{3}\right) \leq h H_{p_{s}}\left(T x_{1}, T x_{2}\right) \text {. }
$$

Again for $h=\frac{1}{\sqrt{k}}$ in (40) we have

$$
p_{s}\left(y_{2}, y_{3}\right) \leq \frac{1}{\sqrt{k}} H_{p_{s}}\left(T x_{1}, T x_{2}\right) .
$$

The other steps follow the similar proof of Theorem (3.1). This complete the proof.

Now, we give an example to illustrate the use of Theorem 3.1.

Example 3.1

Let $X=[0,1]$. Denote the unit interval of real numbers, with the usual convex partial symmetric $p_{s}(x, y)=$ $\|x-y\|$, and a multivalued mapping $T: C \longrightarrow C B^{p_{s}}(X)$ given by

$$
T x= \begin{cases}\frac{x}{2}, & 0 \leq x \leq \frac{2}{3}, \\ -\frac{x}{2}+1, & \frac{2}{3} \leq x \leq 1 .\end{cases}
$$

Define $T: C \longrightarrow 2^{[0,1]}, C=\left[0, \frac{2}{3}\right] \cup\left[\frac{2}{3}, 1\right]$ 
Proof. Since $\partial C$ (boundary of C), $\partial C=\left\{0, \frac{2}{3}, 1\right\}$. Which shows that the fixed point of $T$ are 0 and $\frac{2}{3}$. By using $(i)$ we have

$$
\begin{gathered}
0 \in \partial C \Rightarrow T 0=0 . \\
\frac{2}{3} \in \partial C \Rightarrow T \frac{2}{3}=\frac{1}{3} .
\end{gathered}
$$

Also,

$$
\begin{aligned}
& 1 \in \partial C \Rightarrow T 1=\frac{1}{2} . \\
& \frac{2}{3} \in \partial C \Rightarrow T \frac{2}{3}=\frac{2}{3} .
\end{aligned}
$$

Using (ii), we note that $\{0\}$ and $\left\{0, \frac{2}{3}\right\}$ are bounded sets in $\left(X, p_{s}\right)$. By Lemma 2.1, if $x \in\{0,1\}$, then

$$
\begin{aligned}
x \in \overline{\{0\}} & \Leftrightarrow \operatorname{dist}_{p_{s}}(x,\{0\})=p_{s}(x, x) \\
& \Leftrightarrow p_{s}(x, 0)=|x-0| \\
& \Leftrightarrow x=0 \Leftrightarrow x \in\{0\} .
\end{aligned}
$$

Next,

$$
\begin{aligned}
x \in\left\{0, \frac{2}{3}\right\} & \Leftrightarrow \operatorname{dist}_{p_{s}}\left(x,\left\{0, \frac{2}{3}\right\}\right)=p_{s}(x, x) \\
& \Leftrightarrow \min \left\{p_{s}(x, 0),\left\{0, \frac{2}{3}\right\}\right\} \\
& \Leftrightarrow \min \left\{|x-0|,\left|x-\frac{2}{3}\right|\right\} \\
& \Leftrightarrow x \in\left\{0, \frac{2}{3}\right\} .
\end{aligned}
$$

Hence, $\left\{0, \frac{2}{3}\right\}$ is also closed with respect to partial convex symmetric $p_{s}$.

Note that $T$ is continuous under the partial symmetric $p_{s}$.

Now, to show that the contractive condition of Theorem 3.1 is satisfied, we have the following cases:

Case 1. We now calculate $H_{p_{s}}(T x, T y)$ for $x, y \in\left\{0, \frac{2}{3}\right\}$. Then,

$$
\begin{aligned}
T x & =\left\{0, \frac{1}{3}\right\}, T y=\left\{0, \frac{1}{3}\right\} . \\
H_{p_{s}}(T x, T y) & =H_{p_{s}}\left(\left\{0, \frac{1}{3}\right\},\left\{0, \frac{1}{3}\right\}\right) \\
& =\max \left\{\delta_{p_{s}}(T x, T y), \delta_{p_{s}}(T y, T x)\right\} \\
\delta_{p_{s}}(T x, T y) & =\max \left\{p_{s}(a, T y) ; a \in T x\right\} \\
& =\max \left\{p_{s}(0, T y), p_{s}\left(\frac{1}{3}, T y\right)\right\} \\
p_{s}(0, T y) & =\min \left\{p_{s}(0,0), p_{s}\left(0, \frac{1}{3}\right)\right\} \\
& =\min \left\{0, \frac{1}{3}\right\}=0 . \\
p_{s}\left(\frac{1}{3}, T y\right) & =\min \left\{p_{s}\left(\frac{1}{3}, 0\right), p_{s}\left(\frac{1}{3}, \frac{1}{3}\right)\right\} \\
& =\min \left\{\frac{1}{3}, 0\right\}=0 . \\
\delta_{p_{s}}(T x, T y) & =\max \{0,0\}=0 .
\end{aligned}
$$




$$
\begin{aligned}
\delta_{p_{s}}(T y, T x) & =\max \left\{p_{s}(a, T x) ; a \in T y\right\} \\
& =\max \left\{p_{s}(0, T x), p_{s}\left(\frac{1}{3}, T x\right)\right\} \\
\delta_{p_{s}}(T y, T x) & =\max \left\{0, \frac{1}{3}\right\}=0 . \\
H_{p_{s}}(T x, T y) & =\max \left\{\delta_{p}(T x, T y), \delta_{p}(T y, T x)\right\}, \\
& =\max \{0,0\}=0 .
\end{aligned}
$$

In a similar manner we calculate

$$
\begin{aligned}
p_{s}(x, y) & =p_{s}\left(0, \frac{2}{3}\right)=\frac{2}{3}, \\
p_{s}(x, T x) & =p_{s}\left(0,\left\{0, \frac{1}{3}\right\}\right)=0 \\
p_{s}(y, T y) & =p_{s}\left(\frac{2}{3},\left\{0, \frac{1}{3}\right\}\right)=\frac{1}{3} \\
p_{s}(x, T y) & =p_{s}\left(0,\left\{0, \frac{1}{3}\right\}\right)=0 \\
p_{s}(y, T x) & =p_{s}\left(\frac{2}{3},\left\{0, \frac{1}{3}\right\}\right)=\frac{1}{3}
\end{aligned}
$$

Using the above equations in (8) we obtain

$$
\begin{aligned}
0 & \leq k \max \left\{\frac{2}{3}, 0, \frac{1}{3}, \frac{0+\frac{1}{3}}{2}\right\} \\
& \leq k \max \left\{\frac{2}{3}, 0, \frac{1}{3}, \frac{1}{6}\right\} \\
& \leq \frac{2}{3} k .
\end{aligned}
$$

Equation 8 satisfies with $k=\frac{1}{2}$.

Case 2.

Next, we calculate $H_{p_{s}}(T x, T y)$ for $x \in\left\{0, \frac{2}{3}\right\}, y \in\left\{\frac{2}{3}, 1\right\}$. Then,

$$
\begin{aligned}
T x & =\left\{0, \frac{1}{3}\right\}, T y=\left\{\frac{2}{3}, \frac{1}{2}\right\} . \\
H_{p_{s}}(T x, T y) & =H_{p_{s}}\left(\left\{0, \frac{1}{3}\right\},\left\{\frac{2}{3}, \frac{1}{2}\right\}\right) \\
& =\max \left\{\delta_{p_{s}}(T x, T y), \delta_{p_{s}}(T y, T x)\right\} \\
\delta_{p_{s}}(T x, T y)= & \max \left\{p_{s}(a, T y) ; a \in T x\right\} \\
& =\max \left\{p_{s}(0, T y), p_{s}\left(\frac{1}{3}, T y\right)\right\} \\
& =\max \left\{p_{s}\left(0,\left\{\frac{2}{3}, \frac{1}{2}\right\}\right), p_{s}\left(\frac{1}{3},\left\{\frac{2}{3}, \frac{1}{2}\right\}\right)\right\} \\
\delta_{p_{s}}(T x, T y)= & \max \left\{\frac{1}{2}, \frac{1}{6}\right\}=\frac{1}{2} . \\
\delta_{p_{s}}(T y, T x)= & \max \left\{p_{s}(a, T x) ; a \in T y\right\} \\
& =\max \left\{p_{s}\left(\frac{2}{3}, T x\right), p_{s}\left(\frac{1}{3}, T x\right)\right\} \\
& =\max \left\{p_{s}\left(\frac{2}{3},\left\{0, \frac{1}{3}\right\}\right), p_{s}\left(\frac{1}{3},\left\{0, \frac{1}{3}\right\}\right)\right\} \\
\delta_{p_{s}}(T y, T x) & =\max \left\{\frac{1}{3}, 0\right\}=\frac{1}{3} . \\
H_{p_{s}}(T x, T y) & =\max \left\{\delta_{p}(T x, T y), \delta_{p}(T y, T x)\right\}, \\
& =\max \left\{\frac{1}{2}, \frac{1}{3}\right\}=\frac{1}{2}
\end{aligned}
$$


In a similar manner we calculate

$$
\begin{aligned}
p_{s}(x, y) & =p_{s}(0,1)=1, \\
p_{s}(x, T x) & =p_{s}\left(0,\left\{0, \frac{1}{3}\right\}\right)=0 \\
p_{s}(y, T y) & =p_{s}\left(1,\left\{0, \frac{1}{3}\right\}\right)=0 \\
p_{s}(x, T y) & =p_{s}\left(0,\left\{\frac{2}{3}, 1\right\}\right)=\frac{2}{3} \\
p_{s}(y, T x) & =p_{s}\left(1,\left\{0, \frac{1}{3}\right\}\right)=\frac{2}{3}
\end{aligned}
$$

Using the above equations in (8) we obtain

$$
\begin{aligned}
\frac{1}{2} & \leq k \max \left\{1,0,0, \frac{\frac{2}{3}+\frac{2}{3}}{2}\right\} \\
& \leq k \max \left\{1,0,0, \frac{2}{3}\right\} \\
& \leq k .
\end{aligned}
$$

Equation 8 satisfies with $k=\frac{2}{3}$.

Case 3.

Furthermore, we calculate $H_{p_{s}}(T x, T y)$ for $x, y \in\left\{\frac{2}{3}, 1\right\}$. Let,

$$
\begin{aligned}
T x & =\left\{\frac{2}{3}, \frac{1}{2}\right\}, T y=\left\{\frac{2}{3}, \frac{1}{2}\right\} . \\
H_{p_{s}}(T x, T y) & =H_{p_{s}}\left(\left\{\frac{2}{3}, \frac{1}{2}\right\},\left\{\frac{2}{3}, \frac{1}{2}\right\}\right) \\
& =\max \left\{\delta_{p_{s}}(T x, T y), \delta_{p_{s}}(T y, T x)\right\} \\
\delta_{p_{s}}(T x, T y) & =\max \left\{p_{s}(a, T y) ; a \in T x\right\} \\
& =\max \left\{p_{s}\left(\frac{2}{3}, T y\right), p_{s}\left(\frac{1}{2}, T y\right)\right\} \\
& =\max \left\{p_{s}\left(\frac{2}{3},\left\{\frac{2}{3}, \frac{1}{2}\right\}\right), p_{s}\left(\frac{1}{2},\left\{\frac{2}{3}, \frac{1}{2}\right\}\right)\right\} \\
\delta_{p_{s}}(T x, T y) & =\max \{0,0\}=0 . \\
\delta_{p_{s}}(T y, T x) & =\max \left\{p_{s}(a, T x) ; a \in T y\right\} \\
& =\max \left\{p_{s}\left(\frac{2}{3}, T x\right), p_{s}\left(\frac{1}{2}, T x\right)\right\} \\
& =\max \left\{p_{s}\left(\frac{2}{3},\left\{\frac{2}{3}, \frac{1}{2}\right\}\right), p_{s}\left(\frac{1}{2},\left\{\frac{2}{3}, \frac{1}{2}\right\}\right)\right\} \\
\delta_{p_{s}}(T y, T x) & =\max \{0,0\}=0 . \\
H_{p_{s}}(T x, T y) & =\max \left\{\delta_{p}(T x, T y), \delta_{p}(T y, T x)\right\}, \\
& =\max \{0,0\}=0
\end{aligned}
$$

In a similar manner we calculate

$$
\begin{aligned}
p_{s}(x, y) & =p_{s}(0,1)=1, \\
p_{s}(x, T x) & =p_{s}\left(0,\left\{\frac{2}{3}, \frac{1}{2}\right\}\right)=\frac{1}{2} \\
p_{s}(y, T y) & =p_{s}\left(1,\left\{\frac{2}{3}, \frac{1}{2}\right\}\right)=\frac{1}{3} \\
p_{s}(x, T y) & =p_{s}\left(0,\left\{\frac{2}{3}, \frac{1}{2}\right\}\right)=\frac{1}{2} \\
p_{s}(y, T x) & =p_{s}\left(1,\left\{\frac{2}{3}, \frac{1}{2}\right\}\right)=\frac{1}{3}
\end{aligned}
$$


Using the above equations in (8) we get

$$
\begin{aligned}
0 & \leq k \max \left\{1, \frac{1}{2}, \frac{1}{3}, \frac{\frac{1}{2}+\frac{1}{3}}{2}\right\} \\
& \leq k \max \left\{1, \frac{1}{2}, \frac{1}{3}, \frac{5}{12}\right\} \\
0 & \leq k .
\end{aligned}
$$

Equation 8 satisfies with $k=\frac{1}{2}$.

Which shows that the contraction (8) is satisfied. Therefore, the condition of the Theorem 3.1 is correct; thus, 0 and $\frac{2}{3}$ are two fixed points of $T$.

\section{Conclusions}

The main contribution of this study to fixed point theory is the fixed point result given in Theorem 3.1. This theorem provides the fixed point conditions for a substantial class of non-self mappings on various abstract spaces. This paper, Motivated by the results obtained by Asim et al [16] in partial symmetric metric space and Assad and Kirk in metrically convex metric space. We proved a fixed point theorem for multi-valued non-self mappings in partial symmetric spaces by employing a convex structure using Rhoades type contractions [11], which generalizes some well-known results in the literature. We also provided an illustrative example to support the results.

Conflict of interest: Both authors declare that they have no conflicts of interest.

Research involving human participants and/or animals: The authors declare that there is no human participants and/or animals involved in this research.

Funding: Authors declare that there is no funding available for this research.

\section{References}

[1] S. Banach, Sur les opérations dans les ensembles abstraits et leur application aux équations intégrales. Fund. Math., (1922), no.3, 133-181.

[2] S. B. Nadler, Multi-valued contraction mappings, Pacific Journal of Mathematics, 30(1969), no.2, 475-488.

[3] N. Assad, W. A. Kirk, Fixed point theorems for set-valued mappings of contractive type, Pacific Journal of Mathematics, 43(1972), no.3, 553-562.

[4] L. B. C̀irič, A generalization of Banach contraction principle, Proc. Amer. Math. Soc., 45(1974), no.2, 267-273.

[5] L. B. C̀iric̀ and J. S. Ume, Multi-valued non-self-mappings on convex metric spaces, Nonlinear Analysis: Theory, Methods and Application, 60(2005), no.66, 1053-1063.

[6] N. A. Assad, On some non-self nonlinear contractions, Math. Japon., 33(1988), no.2, 17-26.

[7] N. A. Assad, On some non-self mappings in Banach spaces, Math. Japon., 33(1988), no.2, 501-515.

[8] L. Balog, V. Berinde and M. Păcurar, Approximating fixed points of non-self contractive type mappings in Banach spaces endowed with a graph, Analele Universitatii" Ovidius" Constanta-Seria Matematica, 24(2016), no.2, 27-43.

[9] M. Imdad, A. Ahmad, and S. Kumar, On non-linear non-self hybrid contractions, Rad. Mat., 10(2001), no.2, 233-244.

[10] M. Imdad, and S. Kumar, Rhoades type fixed point theorems for a pair of non-self mappings, Jour. Computers Math. Appl., 46(2003), no. 5-6, 919-927. (MR2020449 (2004h:47082)).

[11] B. E. Rhoades, A fixed point theorem for non-self set-valued mappings, International Journal of Mathematics and Mathematical Sciences, 20(1997), no.1, 9-12.

[12] B. E. Rhoades, A fixed point theorem for a multi-valued non-self mapping, Commentationes Mathematicae Universitatis Carolinae, 37 (1996), no.2, 401-404. 
[13] W. S. Du, E. Karapinar and N. Shahzad, The study of fixed point theory for various multi-valued non-self-maps, In Abstract and Applied Analysis, 2013 Jan 1 (Vol. 2013).

[14] P. Kumam, H. Aydi, E. Karapinar and W. Sintunavarat, Best proximity points and extension of Mizoguchi-Takahashi's fixed point theorems, Fixed Point Theory and Applications, (2013), no. 1, 1-15.

[15] A. Abkar and M. Gabeleh, The existence of best proximity points for multivalued non-self-mappings, Revista de la Real Academia de Ciencias Exactas, Fisicas y Naturales. Serie A. Matematicas, 107(2013), no. 2, 319-325.

[16] M. U. Ali, T. Kamran, and E. Karapinar, A new approach to $(\alpha, \psi)$-contractive non-self multi-valued mappings, Journal of Inequalities and Applications, (2014), no. 1, 1-9.

[17] H. Aydi, A. Felhi and S. Sahmim, Fixed points of multivalued nonself almost contractions in metric-like spaces, Mathematical Sciences, 9 (2015), no. 2, 103-108.

[18] M. U. Ali, T. Kamran and E. Karapinar, Discussion on $\alpha$-Strictly Contractive Nonself Multivalued Maps, Vietnam Journal of Mathematics, 44 (2016), no.2, 441-447.

[19] M. Asim, A. R. Khan and M. Imdad, Fixed point results in Partial Symmetric spaces with an application, Axioms (MDPI), 8(2019), no. 1, 1-15.

[20] S. Matthews, Partial metric topology in Papers on General Topology and Applications, Eighth Summer Conference at Queens College, Eds. S. Andima et al., Annals of the New York Academy of Sciences, 728(1994), 183-197.

[21] W. A. Wilson, On semi-metric spaces, American Journal of Mathematics, 53 (1931), no.2, 361-373.

[22] M. Asim and M. Imdad, Common fixed point results in partial symmetric spaces, TWMS Journal of Applied and Engineering Mathematics,11 (2021), no. 1, 216-227.

[23] M. Asim, H. Aydi and M. Imdad, On Suzuki and Wardowski-Type Contraction Multivalued Mappings in Partial Symmetric Spaces, Journal of Function Spaces, (2021).

[24] S. Kumar and T. Rugumisa, Common fixed points of pair of multi-valued non-self mappings in partial metric spaces, Malaya Journal of Mathematics, 6(2018), no.2, 788-794. https://doi.org/10.26637/MJM0604/0013 